\title{
On an application of Laplace transforms
}

\author{
Murat Duz \\ Department of Mathematics, Faculty of Science, Karabuk University, Karabuk, Turkey
}

Received: 4 August 2015, Accepted: 30 October 2015

Published online: 16 July 2017.

\begin{abstract}
In this study, complex differential equations are solved using laplace transform. Firstly we seperate real and imaginer parts of equation. Thus from one unknown equation is obtained two unknown equation system. Later we obtain laplace transforms of real and imaginer parts of solutions using laplace transform. In the latest we obtain real and imaginer parts of solution using inverse laplace transform.
\end{abstract}

Keywords: Laplace transform, inverse Laplace transform, dirac delta function, complex differential equation.

\section{Introduction}

In real, general solutions of some equations, especially type of elliptic, are not found. Real partial differential equation systems when number of independent variables are even can be transformed to a complex partial differential equations. The solving a complex equation can more easier with complex methods. For example,

$$
u_{x x}+u_{y y}=0
$$

Laplace equation hasn't got general solution in $R^{2}$, but it can be written

$$
u_{z \bar{z}}=0
$$

with the relation

$$
\Delta=\frac{\partial^{2}}{\partial z \partial \bar{z}}
$$

and the solution of the equation is given as

$$
u=f(z)+g(\bar{z})
$$

where $f$ is analytic, $g$ is anti analytic arbitrary functions. A partial differential equation system which has two real dependant and two real independant variables can be transformed to a complex equation. For example,

$$
\begin{aligned}
& u_{x}-v_{y}=0 \\
& u_{y}+v_{x}=0 .
\end{aligned}
$$

Cauchy Riemann system transforms to complex equation

$$
w_{\bar{z}}=0
$$


where $w=u+i v, z=x+i y$. All solutions of this complex equation are analytic functions.

Moreover any order complex differential equation can be transformed to real partial differential equation system which has two unknowns, two independent variables by seperating the real and imaginer parts. The solution of complex equation can be put forward helping solutions of this real system.

In this study, we investigate solutions of first order constant coefficients complex equations with laplace transforms. Laplace transform using several areas of mathematics is a integral transform. We can solve ordinary differential equations, system of ordinary differential equation, integral equations, integro differential equations, difference equations, integro difference equations and also calculate some generalized integrals with laplace transform. Moreover we can use laplace transform in electrical circuits. Therefore we can solve fractional differential equations via laplace transforms[2,3]. Nonlinear differential equations can be solved laplace decomposition method[4].

\section{Basic definitions and theorems}

Definition 1. Let $F(t)$ be a function of $t>0$. Laplace transform of $F(t)$ is defined as

$$
L(F(t))=f(s)=\int_{0}^{\infty} e^{-s t} \cdot f(t) d t
$$

Since integral of (2) is a function of $s$, then we can write $L(F(t))=f(s)$.

Theorem 1. If $F^{(n)}(t)$ is partial continuous, then

$$
L\left(F^{(n)}(t)\right)=s^{n} f(s)-s^{n-1} F(0)-s^{n-2} F^{\prime}(0)-s^{n-2} F^{\prime \prime}(0)-\ldots-F^{(n-1)}(0)
$$

where $L(F(t))=f(s)$.

Theorem 2. Laplace transforms of partial derivatives of $u(x, t)$ are given as follow.

(i) $L\left[\frac{\partial u}{\partial t}\right]=s . U(x, s)-u(x, 0)$

(ii) $L\left[\frac{\partial u}{\partial x}\right]=\frac{\partial U(x, s)}{\partial x}$

where $U(x, s)=L[u(x, t)]$.

\subsection{Complex derivatives}

Let $w=w(z, \bar{z})$ be a complex function. Here $z=x+i y, w(z, \bar{z})=u(x, y)+i \cdot v(x, y)$. First order derivatives according to $z$ and $\bar{z}$ of $w(z, \bar{z})$ are defined as

$$
\begin{aligned}
& \frac{\partial w}{\partial z}=\frac{1}{2}\left(\frac{\partial w}{\partial x}-i \frac{\partial w}{\partial y}\right) \\
& \frac{\partial w}{\partial \bar{z}}=\frac{1}{2}\left(\frac{\partial w}{\partial x}+i \frac{\partial w}{\partial y}\right)
\end{aligned}
$$

\section{Solution of complex differential equations from first order which is constant coeffients}

Theorem 3. Let $A, B, C$ are real constants, $F(z, \bar{z})$ is a polynomial of $z, \bar{z}$ and $w=u+i v$ is a complex function. Then the solution of

$$
A \cdot \frac{\partial w}{\partial z}+B \cdot \frac{\partial w}{\partial \bar{z}}+C \cdot w=F(z, \bar{z})
$$




$$
w(x, 0)=f(x)
$$

is given as

$$
\begin{aligned}
& u=L^{-1}\left[\frac{(A+B) \frac{\partial}{\partial x}\left(2 F_{1}^{*}+(A-B) v(x, 0)\right)+2 C\left(2 F_{1}^{*}+(A-B) v(x, 0)\right)-s(A-B)\left(2 F_{2}^{*}+(B-A) u(x, 0)\right)}{[(A+B) D+2 C]^{2}+s^{2}(A-B)^{2}}\right] \\
& v=L^{-1}\left[\frac{(A+B) \frac{\partial}{\partial x}\left(2 F_{2}^{*}+(B-A) u(x, 0)\right)+2 C\left(2 F_{2}^{*}+(B-A) u(x, 0)\right)-s(B-A)\left(2 F_{1}^{*}+(A-B) v(x, 0)\right)}{[(A+B) D+2 C]^{2}+s^{2}(A-B)^{2}}\right] .
\end{aligned}
$$

Proof.

$$
A \cdot \frac{\partial w}{\partial z}+B \cdot \frac{\partial w}{\partial \bar{z}}+C \cdot w=F(z, \bar{z}) .
$$

We can obtain the following equality using substituting (3), (4) in (5),

$$
A \frac{1}{2}\left(\frac{\partial w}{\partial x}-i \frac{\partial w}{\partial y}\right)+B \frac{1}{2}\left(\frac{\partial w}{\partial x}+i \frac{\partial w}{\partial y}\right)+C w=F_{1}(x, y)+i F_{2}(x, y)
$$

If we write $w=u+i v$ in (6), then we have,

$$
A\left(\frac{\partial u}{\partial x}+i \frac{\partial v}{\partial x}-i \frac{\partial u}{\partial y}+\frac{\partial v}{\partial y}\right)+B \cdot\left(\frac{\partial u}{\partial x}+i \frac{\partial v}{\partial x}+i \frac{\partial u}{\partial y}-\frac{\partial v}{\partial y}\right)+2 C \cdot(u+i v)=2 F_{1}(x, y)+2 i F_{2}(x, y)
$$

If we seperate ( 7) as real and imaginer parts, then following equation system is obtained

$$
\begin{aligned}
& (A+B) \frac{\partial u}{\partial x}+(A-B) \frac{\partial v}{\partial y}+2 C u=2 F_{1}(x, y) \\
& (A+B) \frac{\partial v}{\partial x}+(B-A) \frac{\partial u}{\partial y}+2 C v=2 F_{2}(x, y)
\end{aligned}
$$

If we apply laplace transform to ( 8$),(9)$, then we get the following equalities

$$
\begin{aligned}
& (A+B) \frac{\partial U}{\partial x}+(A-B)(s V-v(x, 0))+2 C U=2 F_{1}^{*} \\
& (A+B) \frac{\partial V}{\partial x}+(B-A)(s U-u(x, 0))+2 C V=2 F_{2}^{*},
\end{aligned}
$$

where $U, V, F_{1}^{*}, F_{2}^{*}$ are laplace transforms of $u, v, F_{1}, F_{2}$ respectively. If $(10),(11)$ are rerugulated, then $(12),(13)$ become

$$
\begin{aligned}
& (A+B) \frac{\partial U}{\partial x}+2 C U+s(A-B) V=2 F_{1}^{*}+(A-B) v(x, 0) \\
& s(B-A) U+(A+B) \frac{\partial V}{\partial x}+2 C V=2 F_{2}^{*}+(B-A) u(x, 0) .
\end{aligned}
$$

Using Cramer rule, we obtaine

$$
\left|\begin{array}{cc}
(A+B) D+2 C & s(A-B) \\
s(B-A) & (A+B) D+2 C
\end{array}\right|=[(A+B) D+2 C]^{2}+s^{2}(A-B)^{2}
$$




$$
\begin{aligned}
& U=\frac{\left|\begin{array}{lc}
2 F_{1}^{*}+(A-B) v(x, 0) & s(A-B) \\
2 F_{2}^{*}+(B-A) u(x, 0) & (A+B) D+2 C
\end{array}\right|}{[(A+B) D+2 C]^{2}+s^{2}(A-B)^{2}} \\
& U=\frac{(A+B) \frac{\partial}{\partial x}\left(2 F_{1}^{*}+(A-B) v(x, 0)\right)+2 C\left(2 F_{1}^{*}+(A-B) v(x, 0)\right)-s(A-B)\left(2 F_{2}^{*}+(B-A) u(x, 0)\right)}{[(A+B) D+2 C]^{2}+s^{2}(A-B)^{2}} \\
& V=\frac{\left|\begin{array}{c}
(A+B) D+2 C 2 F_{1}^{*}+(A-B) v(x, 0) \\
s(B-A) \\
2 F_{2}^{*}+(B-A) u(x, 0)
\end{array}\right|}{[(A+B) D+2 C]^{2}+s^{2}(A-B)^{2}} \\
& V=\frac{(A+B) \frac{\partial}{\partial x}\left(2 F_{2}^{*}+(B-A) u(x, 0)\right)+2 C\left(2 F_{2}^{*}+(B-A) u(x, 0)\right)-s(B-A)\left(2 F_{1}^{*}+(A-B) v(x, 0)\right)}{[(A+B) D+2 C]^{2}+s^{2}(A-B)^{2}} .
\end{aligned}
$$

Followings are obtained from inverse laplace transform of $(12),(13)$.

$$
\begin{aligned}
& u(x, y)=L^{-1}\left[\frac{(A+B) \frac{\partial}{\partial x}\left(2 F_{1}^{*}+(A-B) v(x, 0)\right)+2 C\left(2 F_{1}^{*}+(A-B) v(x, 0)\right)-s(A-B)\left(2 F_{2}^{*}+(B-A) u(x, 0)\right)}{[(A+B) D+2 C]^{2}+s^{2}(A-B)^{2}}\right] \\
& v(x, y)=L^{-1}\left[\frac{(A+B) \frac{\partial}{\partial x}\left(2 F_{2}^{*}+(B-A) u(x, 0)\right)+2 C\left(2 F_{2}^{*}+(B-A) u(x, 0)\right)-s(B-A)\left(2 F_{1}^{*}+(A-B) v(x, 0)\right)}{[(A+B) D+2 C]^{2}+s^{2}(A-B)^{2}}\right]
\end{aligned}
$$

Example 1. Solve the problem

$$
\frac{\partial w}{\partial z}+2 \frac{\partial w}{\partial z}=3 z^{2}+2
$$

with the condition

$$
w(x, 0)=x^{3}+x
$$

Coefficients of equation are $A=1, B=2, C=0$ and $F(z, \bar{z})=3 z^{2}+2$. Real and imaginar parts of $F(z, \bar{z})$ are $F_{1}(x, y)=$ $3 x^{2}-3 y^{2}+2$ and $F_{1}(x, y)=6 x y$. Laplace transforms of $F_{1}(x, y)$ and $F_{2}(x, y)$ are following.

$$
\begin{gathered}
F_{1}^{*}(x, s)=L\left[F_{1}(x, y)\right]=\left(3 x^{2}+2\right) / s-6 / s^{3} \\
F_{2}^{*}(x, s)=L\left[F_{2}(x, y)\right]=6 x / s^{2} .
\end{gathered}
$$

From the above theorem

$$
\begin{aligned}
u(x, y) & =L^{-1}\left[\left(3 \frac{\partial}{\partial x}\left(\left(6 x^{2}+4\right) / s-12 / s^{3}\right)+s\left(12 x / s^{2}+x^{3}+x\right)\right) /\left(9 D^{2}+s^{2}\right)\right] \\
& =L^{-1}\left[\left(48 x / s+s\left(x^{3}+x\right)\right) /\left(9 D^{2}+s^{2}\right)\right] \\
& =L^{-1}\left[\frac{1}{s^{2}\left(1+\frac{9 D^{2}}{s^{2}}\right)}\left(\frac{48 x}{s}+s\left(x^{3}+x\right)\right)\right] \\
& =L^{-1}\left[\frac{1}{s^{2}}\left(\frac{48 x}{s}+s\left(x^{3}+x\right)-\frac{54 x}{s}\right)\right] \\
& =L^{-1}\left[\frac{-6 x}{s^{3}}+\frac{x^{3}+x}{s}\right]=x^{3}+x-3 x y^{2} .
\end{aligned}
$$


Similarly,

$$
\begin{aligned}
v(x, y) & =L^{-1}\left[\frac{3 \frac{\partial}{\partial x}\left(\frac{12 x}{s^{2}}+x^{3}+x\right)-s\left(\left(\frac{\left.6 x^{2}+4\right)}{s}-\frac{12}{s^{3}}\right)\right)}{9 D^{2}+s^{2}}\right] \\
& =L^{-1}\left[\frac{\left(\frac{48}{s^{2}}+3 x^{2}-1\right)}{9 D^{2}+s^{2}}\right] \\
& =L^{-1}\left[\frac{1}{s^{2}\left(1+\frac{9 D^{2}}{s^{2}}\right)}\left(\frac{48}{s^{2}}+3 x^{2}-1\right)\right] \\
& =L^{-1}\left[\frac{1}{s^{2}}\left(-\frac{6}{s^{2}}+3 x^{2}-1\right)\right] \\
& =L^{-1}\left[\frac{-6}{s^{4}}+\frac{3 x^{2}-1}{s^{2}}\right]=-y^{3}+3 x^{2} y-y .
\end{aligned}
$$

Hence

$$
w=u+i v=x^{3}+x-3 x y^{2}+i\left(-y^{3}+3 x^{2} y-y\right)=z^{3}+\bar{z}
$$

Example 2. Solve the following problem

$$
2 \frac{\partial w}{\partial z}-\frac{\partial w}{\partial \bar{z}}=4 z+1
$$

with the condition

$$
w(x, 0)=x^{2}+5 x
$$

Solution 1. Coefficients of equation are $A=2, B=-1, C=0$ and $F(z, \bar{z})=4 z+1$. Thefore

$$
\begin{gathered}
F_{1}^{*}(x, s)=L\left[F_{1}(x, y)\right]=(4 x+1) / s \\
F_{2}^{*}(x, s)=L\left[F_{2}(x, y)\right]=4 / s^{2} \\
u(x, y)=L^{-1}\left[\frac{\frac{\partial}{\partial x}((8 x+2) / s)-3 s\left(8 / s^{2}-3\left(x^{2}+5 x\right)\right)}{D^{2}+s^{2}}\right] \\
=L^{-1}\left[\frac{\frac{8}{s}-\frac{24}{s}+9 s\left(x^{2}+5 x\right)}{D^{2}+9 s^{2}}\right] \\
=L^{-1}\left[\frac{1}{9 s^{2}\left(1+\frac{D^{2}}{9 s^{2}}\right)}\left(\frac{-16}{s}+9 s x^{2}+45 s x\right)\right] \\
=L^{-1}\left[\frac{1}{9 s^{2}}\left(1-\frac{D^{2}}{9 s^{2}}+\frac{D^{4}}{81 s^{4}}-\frac{D^{6}}{729 s^{6}}+\ldots\right)\left(\frac{-16}{s}+9 s x^{2}+45 s x\right)\right] \\
=L^{-1}\left[\frac{1}{9 s^{2}}\left(\frac{-16}{s}+9 s x^{2}+45 s x-\frac{18}{9 s}\right)\right] \\
=L^{-1}\left[-\frac{2}{s^{3}}+\frac{x^{2}+5 x}{s}\right]=x^{2}+5 x-y^{2} .
\end{gathered}
$$


Similarly

$$
\begin{aligned}
v(x, y) & =L^{-1}\left[\frac{\left.\frac{\partial}{\partial x}\left(\frac{8}{s^{2}}-3\left(x^{2}+5 x\right)\right)+3 s(8 x+2) / s\right)}{D^{2}+9 s^{2}}\right] \\
& =L^{-1}\left[\frac{1}{9 s^{2}\left(1+\frac{D^{2}}{9 s^{2}}\right)}(-6 x-15+24 x+6)\right] \\
& =L^{-1}\left[\frac{1}{9 s^{2}}\left(1-\frac{D^{2}}{9 s^{2}}+\frac{D^{4}}{81 s^{4}}-\frac{D^{6}}{729 s^{6}}+\ldots\right)(18 x-9)\right. \\
& =L^{-1}\left[\frac{1}{9 s^{2}}(18 x-9)\right] \\
& =2 x y-y .
\end{aligned}
$$

Consequently

$$
\begin{aligned}
w & =u+i v \\
& =x^{2}+5 x-y^{2}+i(2 x y-y) \\
& =x^{2}+2 i x y-y^{2}+3(x-i y)+2(x+i y) \\
& =z^{2}+3 \bar{z}+2 z .
\end{aligned}
$$

\section{Competing interests}

The authors declare that they have no competing interests.

\section{Authors' contributions}

All authors have contributed to all parts of the article. All authors read and approved the final manuscript.

\section{References}

[1] Yin, F. K., Han, W. Y.and Song, J. Q, "Modified Laplace decomposition method for Lane-Emden Type differential equations", International Jaurnal of Applied Physics and Matematics, 3 (2): 98-102 (2013).

[2] Mohamed, M. A. and Torky, M. S., "Numerical solution of nonlinear system of parial differential equations by the Laplace decomposition method and the padeapproximation", American Journal of Computational Mathematics,3 (3): 175-184 (2013).

[3] Yusufoglu, E., "Numerical Solution of duffing equation by the Laplace decomposition algorithm", Applied Mathematics and Computation,177 (2): 572-580 (2006).

[4] Kazem, S.,'Exact Solution of Some Linear Fractional Differential Equations by Laplace Transform”, International Journal of Nonlinear Science, 16 (2013) No:1 pp. 3-11

[5] Kexue, L., Jigen, P. "Laplace transform and fractional differential equations”, Applied Mathematics Letters, 24 (2011) 2019-2023

[6] Gupta, S., Kumar, D., Singh, J.'Numerical study for systems of fractional differential equations via Laplace transform ", Journal of the Egyptian Mathematical Society, (2015) 23, 256-262 